\title{
Ineffective endovascular treatment of a giant internal carotid artery aneurysm
}

\author{
Paweł Brzegowy ${ }^{1 \mathrm{~B}, \mathrm{DE}, \mathrm{F}}$, Ositadima Chukwu ${ }^{2 \mathrm{E}, \mathrm{F}}$, Katarzyna Ciuk ${ }^{2 \mathrm{E}}$, Andrzej Urbanik ${ }^{1 \mathrm{E}}$, Tadeusz Popiela ${ }^{1 \mathrm{E}}$, \\ Borys Kwinta ${ }^{3 \mathrm{E}}$, Bartłomiej Łasocha ${ }^{4 \mathrm{E}}$
}

'Chair of Radiology, Jagiellonian University Medical College, Krakow, Poland

${ }^{2}$ Students' Scientific Group at the Chair of Radiology, Jagiellonian University Medical College, Krakow, Poland

${ }^{3}$ Department of Neurosurgery, Jagiellonian University Medical College, Krakow, Poland

${ }^{4}$ Department of Imaging Studies, Emergency and Mass-Event Medicine Trauma Centre, University Hospital, Krakow, Poland

\section{Abstract}

\begin{abstract}
Purpose: Despite a growing range of therapeutic possibilities, including various intravascular methods, treating cerebral aneurysms can be still a therapeutic challenge. A growing number of patients previously treated with older techniques require additional therapy. Treatment options as well as their efficiency may be influenced by previous procedures.

Case report: We report a rare case of a giant treatment-resistant aneurysm in a 65-year-old woman. The aneurysm was first diagnosed due to visual disturbances in the right eye. Computed tomography angiography showed large $(20 \times 18 \mathrm{~mm})$ wide neck aneurysm of the right internal carotid artery. The patient was subsequently treated with several methods including coiling with regular stent implantation, two flow diverter stent implantations, and hybrid neurosurgery. Full occlusion was not achieved after any of those procedures. After the last procedure (hybrid neurosurgery) the patient, in vegetative state, was transferred to the intensive care unit and then to the Health and Care Centre.

Conclusions: Discussion focuses on endovascular treatment options after failure of previous treatment such as "stent in stent" technique. We conclude that three subsequent stent implantations are technically possible; however, subsequent procedures are associated with technical difficulties and their effectiveness is questionable. Ventriculoperitoneal shunt may influence the outcome of flow diversion therapy.
\end{abstract}

Key words: endovascular treatment, flow diverter, stent-in-stent technique, intracranial aneurysm

\section{Introduction}

The prevalence of intracranial aneurysms varies between 0.5 and $4 \%$, depending on the methodology of the study [1]. However, most of them are asymptomatic [1]. Giant intracranial aneurysms are defined by a diameter greater than $25 \mathrm{~mm}$. They are relatively rare. They constitute $2-5 \%$ of all aneurysms $[2,3]$. There are several strategies to treat giant intracranial aneurysms, but they are still a therapeutic challenge with the risk of perioperative complications and recurrence. Treatment options include neurosurgical techniques (clipping, bypass) and a wide range of endovascular procedures (parent vessel occlusion, coiling, flow diverter stents, and more complicated devices such as p-conus used in particular cases).

Nowadays flow diversion with additional coiling is preferred in giant aneurysm cases. However, the rapid development of new options has left us in the situation

Correspondence address:

Ositadima Chukwu, Students' Scientific Group at the Chair of Radiology, Jagiellonian University Medical College, 19 Kopernika St., 31-501 Krakow, Poland,

e-mail: stdmchkw@gmail.com

Authors' contribution:

A Study design · B Data collection · C Statistical analysis · D Data interpretation · E Manuscript preparation · F Literature search · G Funds collection 
in which a growing number of patients previously treated with older methods need additional therapy. The safety and efficiency of flow diverter stents used after other types of treatment is still being investigated.

We report a rare case of a giant recurrent aneurysm that was subsequently treated several times with techniques including coiling with regular stent implantation, two flow diverter stent implantations, and hybrid neurosurgery. The history of use of these various methods enables us to see the progressive change of the paradigm in treating intracranial aneurysms.

We decided to describe this case because it is the first one of totally ineffective endovascular treatment with flowdiverted stents in our centre.

\section{Case report}

A 65-year-old woman with visual disturbances in the right eye was diagnosed using computed tomography angiography (CTA) with a large unruptured aneurysm of a right internal carotid artery. The aneurysm was localised in the C6 segment of the right internal carotid artery (RICA). The dimensions of the aneurysm determined by CTA were $20 \times 18 \mathrm{~mm}$; the neck was $8 \mathrm{~mm}$ wide.

\section{Treatment}

\section{First procedure - stent-assisted coiling}

In August 2012 the patient was qualified for endovascular treatment. After a standard five days of oral dual antiplatelet preparation (ASA $150 \mathrm{mg}$ and clopidogrel $75 \mathrm{mg}$ ) endovascular embolisation - stent-assisted coiling (with a Leo plus stent) - was performed. Control digital subtraction angiography (DSA) per-

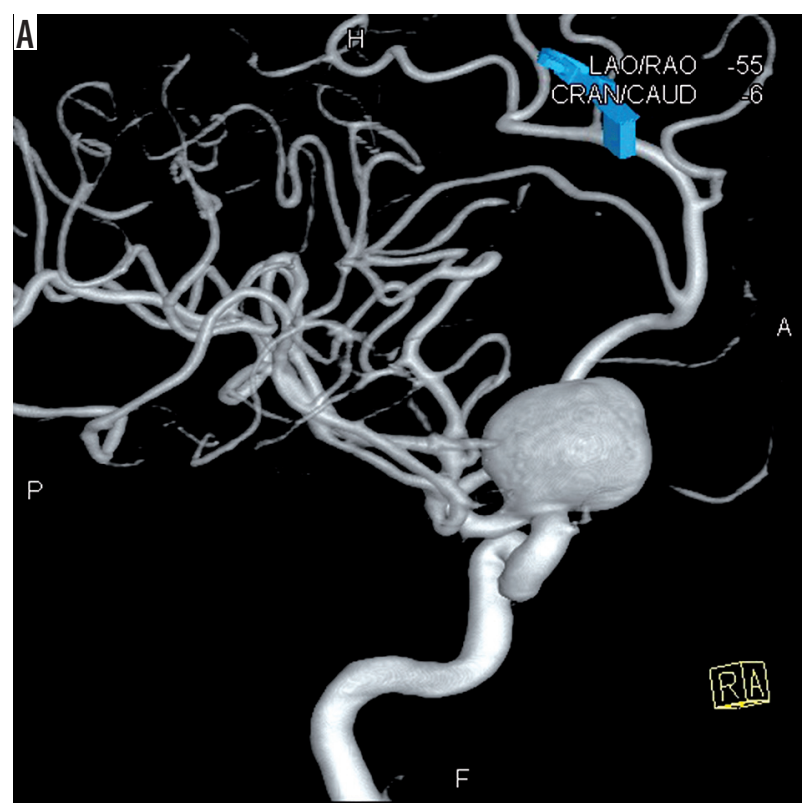

formed after the procedure showed occlusion of the aneurysm with partial filing of the base of a sac - Raymond-Roy Occlusion Classification (RROC) IIIa. The patient was then discharged home from hospital with administered dual-antiplatelet therapy for six months. Control DSA was scheduled one year after the procedure.

\section{Second procedure - flow diverter}

Twelve months later, in August 2013, a control DSA revealed recanalisation of the aneurysmal sac (RROC IIIb) and enlargement of the whole aneurysm to dimensions up to $34 \times 25 \mathrm{~mm}$. A complementary endovascular procedure was performed - additional coils were placed into the aneurysmal sac and a flow-diverter stent (FRED) was placed into the previously implanted Leo stent. Control angiography showed a small filling with contrast medium (CM) stagnation at the base of the aneurysmal sac (RROC IIIa). The patient left the hospital in a good clinical and neurological condition with persistent visual disturbance of the right eye.

\section{Third procedure - ventriculoperitoneal shunt}

In April 2016 the patient underwent a procedure of placement of a ventriculoperitoneal shunt due to hydrocephalus in another health care centre.

\section{Fourth procedure - second flow diverter}

In July 2016 the patient was admitted to the hospital due to worsening of neurological condition. She was psychomotorically slowed down, she had paresis of left lower limbs (intensity 4/5 in Lovett scale), and central paresis of the left facial nerve. Meningeal signs were negative.

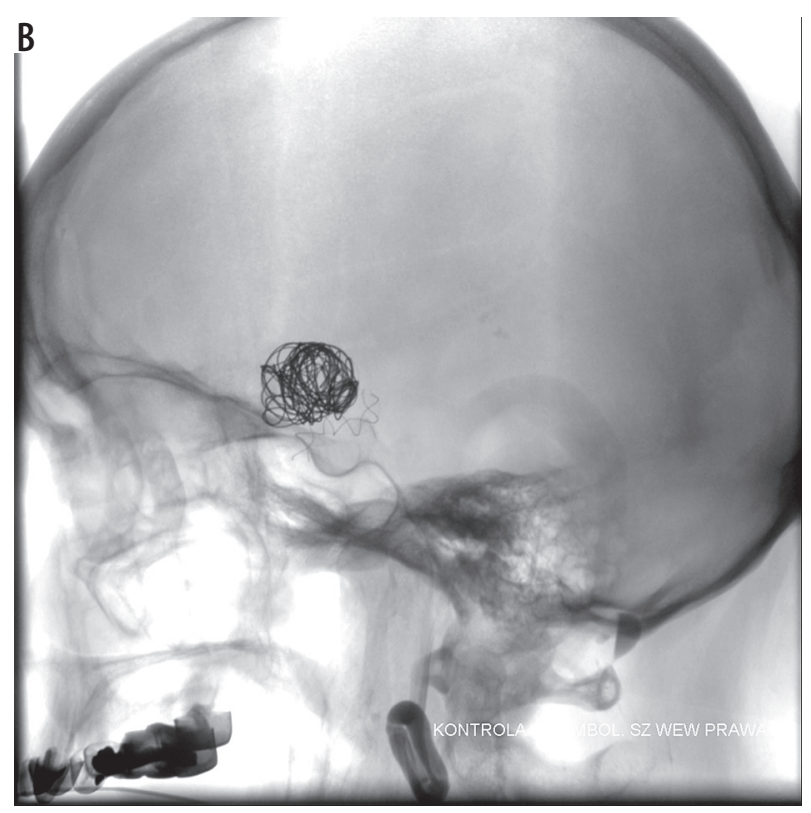

Figure 1. First procedure. A) Pre-treatment state. Aneurysm located in (6 segment of right internal carotid artery. 3D rotational angiography. B) Control angiography performed during procedure. Stent and coils are visible. Digital subtraction angiography 
A

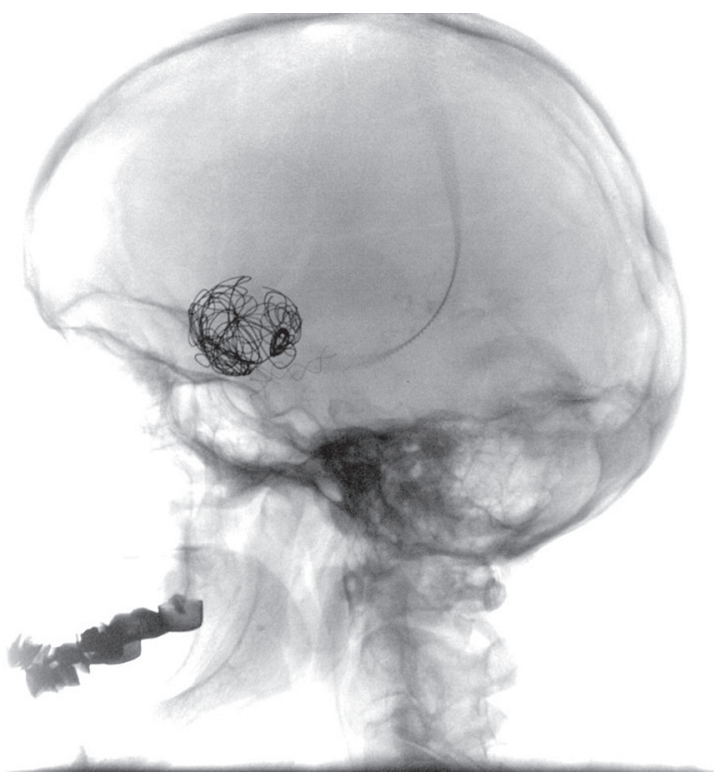

B

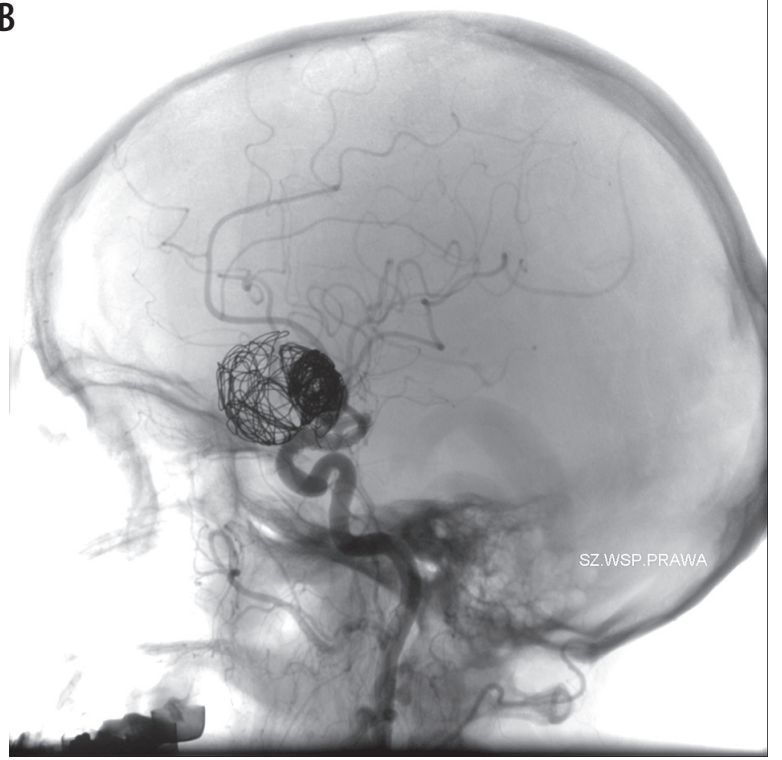

Figure 2. Second procedure. A) Before the procedure: we can see displacement of coils and enlargement of aneurysm. B) After the procedure: additional coils are visible, base of sack is slightly filed with contrast
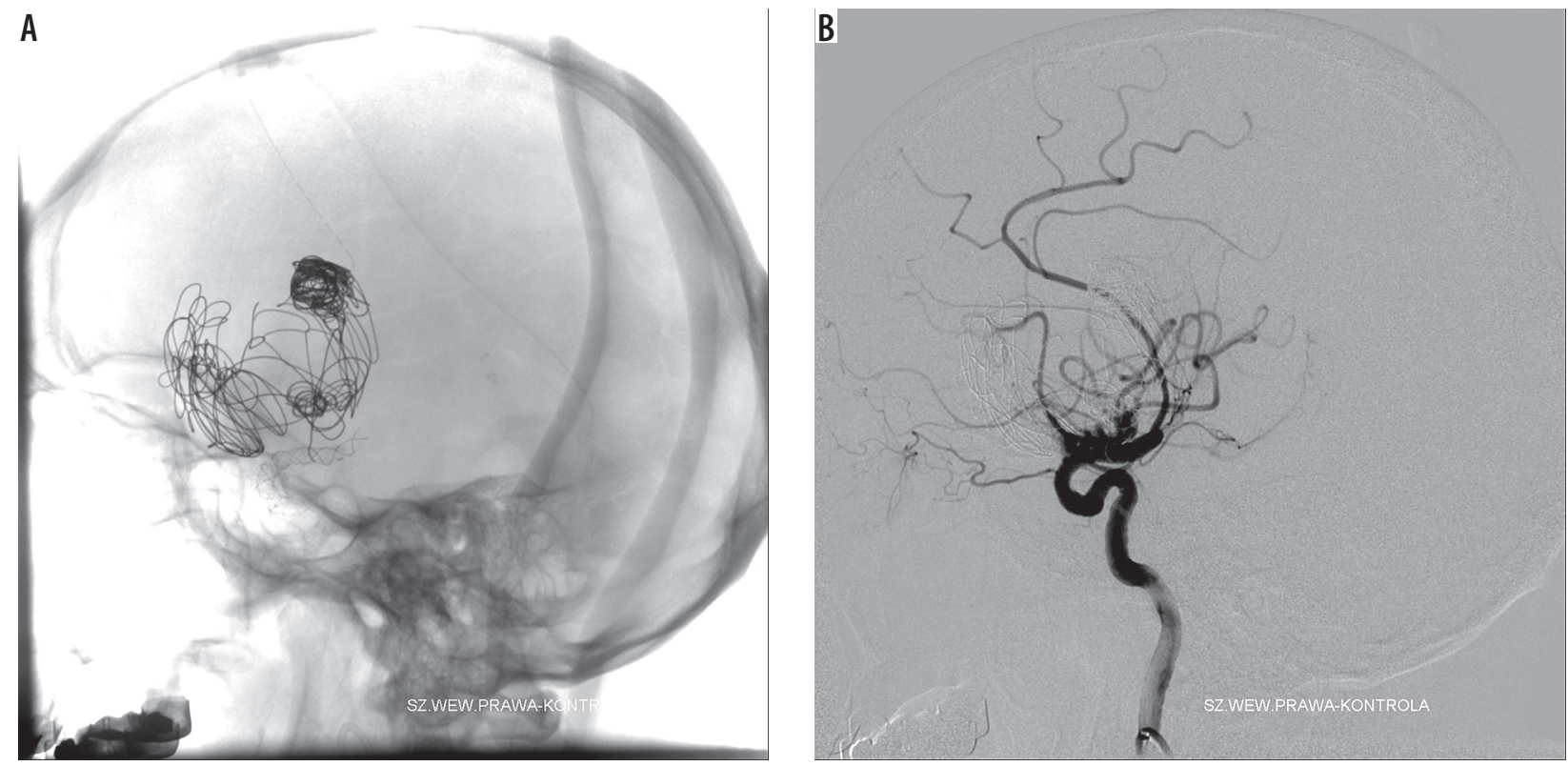

Figure 3. Fourth procedure. A) Before the procedure: two previously placed stents are visible as well as coils. B) After the procedure: partial filing of the base of a sack is still present

Control cerebral DSA showed recanalisation of the previously treated aneurysm. The aneurysm enlarged up to $42 \times 34 \mathrm{~mm}$. Slow inflow of $\mathrm{CM}$ to the base of the aneurysmal sack was present. The area filled with CM measured approximately $15 \times 4 \mathrm{~mm}$. Previously implanted stents were not dislocated and fully covered the aneurysmal neck. Previously deployed coils were spread and dislocated. The patient was again qualified for endovascular treatment with the use of another flow diverter.

The procedure was performed using a tri-axial system in the RICA. A long $6 \mathrm{~F}$ introducer sheath, $6 \mathrm{~F}$ guiding catheter (Chaperon), and intermediate catheter (Sofia $5 \mathrm{~F}$ ) were used to pass kinking of the extracranial segment of the RICA. Passing through the lumen of the two previous stents required numerous try-outs and stabilisation of the tri-axial system. A SILK plus flow-diverted stent was implanted to segment 6 of the RICA into the two previously placed stents, covering the aneurysmal neck. In control DSA the stent completely covered the neck of the aneurysm, slow inflow of contrast into aneurysmal sack was present, and blood flow through right hemisphere was maintained. There were no complications during the procedure. The patient was rehabilitated and discharged home.

\section{Fifth procedure - hybrid neurosurgery}

Three months later the patient was admitted to hospital with symptoms of intracranial bleeding. CT revealed 


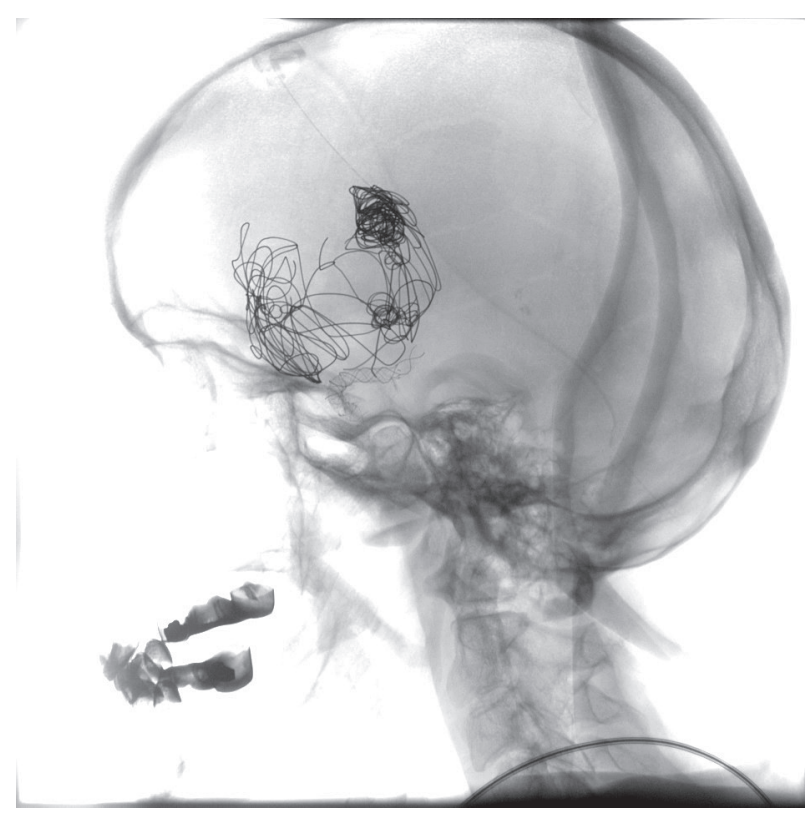

Figure 4. State before hybrid operation. Three stents placed in light of vessel are visible

intracerebral bleeding in the right hemisphere, blood in ventricles, with brain oedema. Rupture of the aneurysm was believed to be the source of bleeding. The patient was qualified for hybrid vascular intervention.

From femoral access, a guiding catheter was placed into the RICA. A balloon catheter (Scepter) was placed in the RICA inside stents at the level of the aneurysmal sac, its position was confirmed using DSA. After that the surgical part of the procedure was performed. During evacuation of the aneurysmal sack content, the balloon was inflated four times to prevent bleeding. After clipping, control DSA was performed, and contrast medium flow inside the right hemisphere was confirmed. After the procedure the patient, in vegetative state, was transferred to the Intensive Care Unit and then to the Health and Care Centre.

\section{Discussion}

In the described case, initially the patient was treated with stent-assisted coiling, which was considered the optimal therapy at that time because in our centre flow-diverter stents were not available [4].

A wide neck of aneurysms was causing a risk of coil mass prolapse into the parent vessel. A stent secures the coil mass and enables doctor to increase the packing density of the coils. The main advantage of flow-diverter stents in comparison to regular stents is the reduction of blood flow entering the aneurysm. Initial treatment was unsuccessful and did not stop the growth of the aneurysm. The failure of the first procedure might have been caused by using a regular stent, not a flow-diverter stent, which is currently preferred.

However, new therapeutic challenges have arisen. At the time of the second procedure flow diverter therapy was considered as an optimal therapy. The second stent had to be telescoped inside the previous one. Several studies have shown the safety of the 'stent-in-stent' technique $[5,6]$, although its exact risk is yet to be established. The efficiency of the second procedure might have been affected by the implantation of a ventriculoperitoneal shunt. Decreased volume of cerebral fluid might have caused a gradient of pressure, which may have caused further enlargement of the aneurysm. Recurrence of aneurysms is possible even after complete occlusion [7].

Application of the third stent into the vessel was problematic. Technical difficulties were overcome by using a thinner flow diverter, stabilising long sheath, and a micro catheter. The number of consecutive 'stent-in-stent' procedures is limited not only by the size of the vessel and previously used stents but also by the technical abilities of the person performing the procedure. The risk of complications following subsequent 'stent-in-stent' applications has not been established yet, and previous studies have shown that the presence of a pre-existing stent may influence the effectiveness of the treatment [8]. The ventriculoperitoneal shunt was still present, so it might have influenced the outcome of the treatment.

The reason why the flow diverters failed to secure the aneurysm is unclear. Several factors are proven to decrease the occlusion rate after flow-diverter therapy, such as fusiform aneurysm morphology, decreased dome-neck ratio, and the presence of a pre-existing stent [2]. Technical complications, such as malposition to the parent vessel wall, inadequate coverage of the aneurysmal neck, and incorporation of a branch vessel into the aneurysm fundus, are also postulated as possible mechanisms of treatment failure [2, 9]. Implantation of a ventriculoperitoneal shunt and the presence of the first stent are probable causes in this case.

After the failure of occlusion using endovascular stents the treatment options are limited by the presence of the stents $[2,10]$. Deployment of the next flow diverter or surgery are plausible [2]. In this case endovascular treatment was preferred due to the patient's age and comorbidities.

The patient was initially disqualified from neurosurgery due to their age and widespread atherosclerosis. Surgical treatment due to high risk of complications was considered the last rescue option. Surgery was performed after a large intracranial bleeding. It is likely that worsening of the patient's condition can be attributed more to the previous bleeding than to the procedure itself. Another treatment option - occlusion of the parent vessel - was contraindicated due to lack of collateral circulation, which was found during the DSA $[11,12,13]$.

During flow diverter implantation no direct procedure-related complications occurred. There were no maldeployments of flow diverters including incomplete expansion, migration, and prolapse. Thromboembolic and ischaemic complications as well as parent vessel injury were also absent. It is unclear if haemorrhage from an aneurysm can be attributed to endovascular treatment. The risk of the rupture in an aneurysm of this size was 
initially high, so haemorrhage could be attributed to lack of efficiency of the treatment.

Failure of the treatment may be influenced by a number of factors, many of which are not dependent on the abilities of the physicians. However, even after initial defeat, many treating options are still available. The choice of the method must be based on the patient's condition.

\section{Conclusions}

We conclude that three subsequent stent implantations are technically possible; however, subsequent procedures are associated with technical difficulties, and their effectiveness is questionable. In the treatment of giant aneurysms flow-diverter stents should be preferred over regular stents. If there is hydrocephalus or high risk of hydrocephalus, surgery should be considered because flow diversion with additional coiling may not be effective.

\section{Conflict of interest}

The authors declare no conflict of interest.

\section{References}

1. Keedy A. An overview of intracranial aneurysms. Mcgill J Med 2006; 9: 141-146.

2. Al-Mufti F, Amuluru K, Cohen E, et al. Rescue Therapy for Procedural Complications Associated With Deployment of Flow-Diverting Devices in Cerebral Aneurysms. Oper Neurosurg 2018; 15: 624-633.

3. Lonjon M, Pennes F, Sedat J, et al. Epidemiology, genetic, natural history and clinical presentation of giant cerebral aneurysms. Neurochirurgie 2015; 61: 361-365.

4. Lv X, Jiang C, Li Y, et al. Treatment of Giant Intracranial Aneurysms Interv Neuroradiol 2009; 15: 135-144.

5. Aydin K, Barburoglu M, Sencer S, et al. Flow diversion with low-profile braided stents for the treatment of very small or uncoilable intracranial aneurysms at or distal to the circle of Willis. Am J Neuroradiol 2017; 38: 2131-2137.

6. Cohen J, Gomori J, Moscovici S, et al. Successful endovascular treatment of a growing megadolichoectasic vertebrobasilar artery aneurysm by flow diversion using the "diverter-in-stent" technique. J Clin Neurosci 2012; 19: 166-170.
7. Trivelato FP, Ulhôa AC, Rezende MT, et al. Republished: Recurrence of a totally occluded aneurysm after treatment with a pipeline embolization device. J Neurointerv Surg 2019; 11: e5.

8. Lang ST, Assis Z, Wong JH, et al. Rapid delayed growth of ruptured supraclinoid blister aneurysm after successful flow diverting stent treatment. BMJ Case Rep 2016; 2016: bcr2016012506.

9. Shapiro M, Becske T, Nelson PK. Learning from failure: persistence of aneurysms following pipeline embolization. J Neurosurg 2017; 126: 578-585.

10. Gressot LV, Patel AJ, Srinivasan VM, et al. An intraoperative look at failure of flow diversion: when additional or alternative treatments should be considered. World Neurosurg 2016; 93: 486.e7-486.e12.

11. Benson CM, Pelz DM, Lownie SP. Parent artery occlusion: a wellestablished technique. AJNR Am J Neuroradiol 2015; 36: E53.

12. Cui L, Peng Q, Ha W. et al. Parent artery occlusion for intracranial aneurysms. Interv Neuroradiol 2009; 15: 309-315.

13. Nishi H, Ishii A, Satow T, et al. Parent artery occlusion for unruptured cerebral aneurysms: results of the japanese registry of neuroendovascular therapy 3. Neurol Med Chir (Tokyo) 2019; 59: 1-9. 